ISSN electrónico: 2602-8069

\title{
VALORACIÓN DEL APRENDIZAJE COOPERATIVO SEGÚN LA ESCALA DE APLICACIÓN CLAS
}

\section{Assessment of Cooperative Learning according to the CLAS Application Scale}

Alex Mora Benítez
Universidad UTE
bralexmb@yahoo.com.mx

Fecha de recepción del artículo: 1812/2019

Fecha de aceptación definitiva: 19/02/2020 


\section{RESUMEN}

El presente estudio de tipo experimental, realizado con los estudiantes de nivelación pertenecientes al ámbito universitario, muestra los resultados obtenidos a partir de la implementación del Aprendizaje Cooperativo (AC) y la valoración del mismo por medio del instrumento de medición CLAS. Las siete dimensiones del instrumento permitieron a los estudiantes valorar el uso del AC y dieron a conocer que la implementación del AC tuvo una aceptación del $93.46 \%$, siendo la tutoría realizada por el docente durante la aplicación del AC la variable más importante, lo cual denota la valía de la participación docente en el aprendizaje cooperativo. A partir de esta iniciativa se abren posibilidades de implementar en las aulas metodologías constructivas y participativas en beneficio de los procesos de aprendizaje tomando como protagonistas tanto a los educandos como a los docentes.

Palabras clave: aprendizaje, proceso de aprendizaje, evaluación de la educación, investigación pedagógica, valoración del estudiante.

\section{ABSTRACT}

The present experimental study, conducted on leveling students belonging to the university field, shows the results obtained from the implementation of Cooperative Learning (CA) and its assessment through the CLAS measurement instrument. The seven dimensions of the instrument allowed students to assess the use of the AC and made it known that the implementation of the AC had an acceptance of $93.46 \%$, with the Tutoring carried out by the teacher during the application of the AC being the most important dimension, which denotes the value of teacher participation in cooperative learning. From this initiative, possibilities are opened to implement constructive and participatory methodologies in the classroom for the benefit of the learning processes taking as protagonists both the students and the teachers.

Keywords: Learning, Learning Process, Education Evaluation, Pedagogical Research, Student Assessment. 


\section{INTRODUCCIÓN}

El desarrollo humano está relacionado directamente con el aprendizaje, cada nuevo invento, cada posibilidad, cada solución que permite abordar un problema requiere llevar a cabo acciones relacionadas con el aprender, es una necesidad humana infinita. Visto desde la educación el aprendizaje se convierte en el principal objetivo del acto de educar, y son varios los paradigmas desde donde se puede iluminar el mencionado acto. Actualmente uno de los paradigmas del proceso de aprendizaje es aquel que coloca como protagonista al educando, quien se conforma y construye con el apoyo del educador.

El Aprendizaje Cooperativo (AC) se presenta como una estrategia pedagógica útil para desarrollar múltiples habilidades y competencias sociales, según Atxurra, Villardón-Gallego y Calvete (2015): " El AC es una metodología de aprendizaje centrada en el estudiante, que cuando se aplica eficazmente, favorece el desarrollo de determinadas competencias útiles en la vida profesional y social", a diferencia del común "trabajo en grupo", los estudiantes son protagonistas de su aprendizaje, sin descartar el trabajo del docente, tomando en cuenta que la eficacia del AC es el resultado de la forma como el docente estructura la cooperación y asegura las condiciones necesarias para lograr este resultado (Atxurra, C. et al., 2015), es decir la participación de los estudiantes es medular, y la del docente de igual forma en las orientaciones que proporciona acerca de la metodología.

En el ámbito ecuatoriano se ha encontrado algunas aplicaciones del AC, como es el caso de la enseñanza de los Estudios Sociales a nivel de bachillerato (Placencia y Díaz, 2015), además ha sido utilizado para la enseñanza del idioma inglés en algunas escuelas públicas (Loor, Palma, Saltos y Bolívar, 2018), así como en la educación en valores por medio de la propuesta didáctica de Cisneros (2018) para los estudiantes de Primer año de Bachillerato, entre otras, siendo estas, contribuciones importantes en cuanto al AC. El Aprendizaje Cooperativo también se lo puede utilizar en la Educación Superior, siendo aplicable a todas las carreras, en las cuales los estudiantes requieren desarrollar el trabajo en equipo, el pensamiento crítico y practicar varias habilidades sociales que les permitirán generar un adecuado desenvolvimiento en su entorno de trabajo, como se muestra en esta investigación, al ser utililizado en estudiantes de nivelación de Ciencias de la Salud.

Además, el AC ha sido utilizado en muchas investigaciones, actualmente, constituye una metodología innovadora que puede ayudar a resolver los problemas más acuciantes en el ámbito educativo, como: el fracaso escolar, la falta de motivación, las relaciones profesores alumno, el maltrato entre iguales y, por supuesto, el tratamiento de la multiculturalidad en el aula (Díaz-Aguado, 2003, citado en León, 2014), inicialmente esta metodología apuntaba a lograr mejores resultados en los temas académicos, sin embargo, varios estudios muestran que se presentaban actitudes positivas y solidarias entre los participantes, quienes actuaban de forma cooperativa no solo en la producción de saberes sino en cuanto al accionar basado en valores, emociones positivas, integración y adaptación escolar (León, 2014).

El trabajo del docente es de vital importancia en el desarrollo del aprendizaje cooperativo, Johnson y Johnson (1999) sostienen que se logran tres metas fundamentales con el AC, en primera instancia, ayuda a elevar el rendimiento académico de los estudiantes incluyendo a los que tienen dotes especiales como aquellos que presentan dificultades para aprender; en segundo lugar, los estudiantes aprenden a establecer relaciones positivas entre ellos, consolidando las bases de una comunidad de aprendizaje basada en la diversidad; y tercero, el AC proporciona a los estudiantes experiencias para lograr un saludable desarrollo social, psicoló- 
gico y cognitivo, estos elementos hacen del AC una metodología de construcción social en la que se alcanzan aprendizajes que traspasan las expectativas cognitivas.

Se descarta la competitividad y la gran producción debido a que su base de funcionamiento es la fortificación de un equipo de trabajo de alto desempeño acompañado por la participación activa del docente que requiere de su ingenio para organizar y facilitar el aprendizaje, es decir, el rol docente no es el de un observador pasivo expectante, sino de un actor promotor de experiencias con materiales necesarios para que los estudiantes cooperen, a distinción de los métodos tradicionales de enseñanza que buscan llenar las mentes de sus oyentes como si fuesen recipientes vacíos, el aprendizaje cooperativo está basado en la experiencia y la solidaridad (Johnson y Johnson, 1999).

EI AC cuenta con múltiples variables como son: la composición del grupo que considere la heterogeneidad de sus integrantes, la estructura del grupo que conlleva el tamaño y grado de cohesión entre sus miembros, el ambiente físico y lugar de las sesiones así como el mobiliario y su distribución espacial, el ambiente organizacional en donde el papel del educador requiere potenciar sus habilidades sociales de apoyo y acompañamiento (Fernández, 2010), estos elementos deben ser tomados en cuenta por parte del docente previo al proceso de AC. Ya en el proceso del AC, el mismo autor manifiesta: el AC incrementa la productividad individual y colectiva, fomenta el pensamiento crítico, la creatividad, el actuar autónomo, la vivencia de valores esenciales de la convivencia social como el respeto, aceptación del otro, solidaridad, responsabilidad, autoestima y paz, la comunicación es fluida y abierta para conocer el punto vista de los demás, lo que permite visualizar la realidad desde diferentes focos de observación (Fernández, 2010). Dando a conocer una relación directa entre el aprendizaje cooperativo y el instrumento de medición CLAS con sus dimensiones respectivas. EI AC significa cooperar y aprender, hacerse mutuamente responsables de los logros y también de los desaciertos, todos los estudiantes llegan a sus objetivos y aprenden a sostenerse, estos elementos son valorados a través de las dimensiones del CLAS.

\section{METODOLOGÍA}

En el estudio participaron 63 estudiantes (Ver tabla 2) de nivelación que cursaron la materia de Bases de la Comunicación, 25 fueron hombres y 38 mujeres, en porcentajes representan el $38.68 \%$ de hombres y $60.32 \%$ de mujeres; del total de estudiantes el 23.81 $\%$ provenían de colegios públicos y el $76.19 \%$ de colegios privados; cabe señalar que el $19.05 \%$ de los estudiantes tenían beca y el $80.95 \%$ no eran becarios, finalmente el $11.11 \%$ del total de participantes tenían menos de 18 años, mientras que el $88.89 \%$ superaban los 18 años, con estas consideraciones transformadas en criterios se conformaron 12 equipos de trabajo.

Tabla 2. Características del grupo de estudio

\begin{tabular}{|l|l|l|}
\hline \multicolumn{3}{|c|}{ Total 63 estudiantes } \\
\hline Género & 25 hombres $-38.68 \%$ & 38 mujeres $-60.32 \%$ \\
\hline Colegio de origen & $23,81 \%$ Públicos & $76,19 \%$ Privados \\
\hline Origen de matrícula & $19.05 \%$ Becados & $80.95 \%$ No becados \\
\hline Rango edad & $11.11 \%>$ a 18 años & $88.89 \%<18$ años \\
\hline
\end{tabular}


Se toman como afirmativas las respuestas "De acuerdo" y "Muy de acuerdo", al sumarse las respuestas se obtiene el porcentaje con respecto al total en cada una de las dimensiones, posteriormente se obtiene el promedio de cada una de las dimensiones, como se muestra en la tabla 3:

Tabla 3. Porcentajes por pregunta - Univariante

\begin{tabular}{|c|c|c|c|c|c|}
\hline Evaluación & DA & MDA & Total & $\%$ & Promedio \\
\hline 42 & 20 & 27 & 47 & 74.6 & 88,4 \\
\hline 34 & 34 & 17 & 51 & 81.0 & \\
\hline 3 & 30 & 30 & 60 & 95.2 & \\
\hline 15 & 28 & 28 & 56 & 88.9 & \\
\hline 29 & 19 & 42 & 61 & 96.8 & \\
\hline 22 & 10 & 49 & 59 & 93.7 & \\
\hline Heterogeneidad & DA & MDA & Total & $\%$ & Promedio \\
\hline 1 & 24 & 36 & 60 & 95.2 & 93.7 \\
\hline 9 & 23 & 36 & 59 & 93.7 & \\
\hline 16 & 25 & 33 & 58 & 92.1 & \\
\hline 23 & 23 & 36 & 59 & 93.7 & \\
\hline Habilidades Soc. & DA & MDA & Total & $\%$ & Promedio \\
\hline 7 & 26 & 35 & 61 & 96.8 & 94.3 \\
\hline 2 & 30 & 32 & 62 & 98.4 & \\
\hline 10 & 24 & 34 & 58 & 92.1 & \\
\hline 35 & 30 & 25 & 55 & 87.3 & \\
\hline 30 & 25 & 35 & 60 & 95.2 & \\
\hline 24 & 19 & 43 & 62 & 98,4 & \\
\hline 17 & 10 & 48 & 58 & 92,1 & \\
\hline Interacción & DA & MDA & Total & $\%$ & Promedio \\
\hline 43 & 23 & 38 & 61 & 96.8 & 96,8 \\
\hline 25 & 19 & 43 & 62 & 98.4 & \\
\hline 11 & 15 & 46 & 61 & 96.8 & \\
\hline 18 & 19 & 41 & 60 & 95.2 & \\
\hline $\begin{array}{l}\text { Interdependencia } \\
\text { positiva }\end{array}$ & DA & MDA & Total & $\%$ & Promedio \\
\hline 36 & 28 & 22 & 50 & 79.4 & 91.7 \\
\hline 26 & 24 & 31 & 55 & 87.3 & \\
\hline 19 & 17 & 42 & 59 & 93,7 & \\
\hline 38 & 21 & 38 & 59 & 93,7 & \\
\hline 31 & 29 & 29 & 58 & 92,1 & \\
\hline 40 & 21 & 41 & 62 & 98,4 & \\
\hline 32 & 23 & 34 & 57 & 90.5 & \\
\hline 8 & 27 & 32 & 59 & 93.7 & \\
\hline 12 & 16 & 45 & 61 & 96,8 & \\
\hline
\end{tabular}




\begin{tabular}{|c|c|c|c|c|c|}
\hline Reflexión Grupal & DA & MDA & Total & $\%$ & Promedio \\
\hline 5 & 27 & 27 & 54 & 85.7 & 91.6 \\
\hline 13 & 30 & 30 & 60 & 95.2 & \\
\hline 37 & 34 & 25 & 59 & 93.7 & \\
\hline 4 & 24 & 36 & 60 & 95.2 & \\
\hline 27 & 35 & 21 & 56 & 88.9 & \\
\hline 20 & 29 & 27 & 56 & 88.9 & \\
\hline 6 & 31 & 28 & 59 & 93.7 & \\
\hline Tutoría & DA & MDA & Total & $\%$ & Promedio \\
\hline 21 & 17 & 44 & 61 & 96.8 & 97.7 \\
\hline 44 & 21 & 40 & 61 & 96.8 & \\
\hline 41 & 25 & 37 & 62 & 98.4 & \\
\hline 14 & 22 & 40 & 62 & 98.4 & \\
\hline 39 & 15 & 45 & 60 & 95.2 & \\
\hline 33 & 24 & 38 & 62 & 98.4 & \\
\hline 28 & 17 & 46 & 63 & 100.0 & \\
\hline
\end{tabular}

Según los datos de la tabla 3 se observa que:

La dimensión Evaluación está formada por seis ítems 42, 34, 3, 15, 29 y 22, con un $74 \%$ en el ítem 42 que representa la sumatoria más baja y el ítem 29 con un $96.8 \%$ con el porcentaje más elevado. La Heterogeneidad como segunda dimensión está sostenida por los ítems 1, 9, 16 y 23, así el ítem 16 se muestra con el $92.1 \%$ como el porcentaje más bajo, mientras que el ítem 1 adquiere el $95.2 \%$ como porcentaje más alto. La dimensión Habilidades Sociales se conforma por los ítems 7, 2, 10, 35, 30, 24 y 17, el porcentaje más bajo se refiere al ítem 35 con el $87.3 \%$, no así los ítems 2 y 24 que muestran el mayor porcentaje con un $98.4 \%$.

La dimensión de Interacción está sostenida por los ítems 43, 25, 11 y 18, teniendo al ítem 18 con el porcentaje más bajo $95.2 \%$, mientras el ítem 25 se muestra con un $98.4 \%$ como el porcentaje más elevado. La Interdependencia Positiva es la que mayor cantidad de ítems tiene: $36,26,19,38,31,40,32,8$ y 12, siendo el ítem 36 el que menor porcentaje tiene con el $79.4 \%$ y el ítem 40 con el $98.4 \%$ como el porcentaje más elevado. La Reflexión Grupal se sostiene en los ítems: $5,13,37,4,27,20$ y 6 , teniendo el ítem 5 el porcentaje más bajo con $85.7 \%$, y los ítems 13 y 4 con el 95.2 como porcentajes más altos. Finalmente, la dimensión de Tutoría formada por los ítems 21, 44, 41, 14, 39, 33 y 28, muestran el porcentaje más bajo en el ítem 39 con el $95.2 \%$ y el ítem 28 es el ítem con porcentaje perfecto de $100 \%$.

Los datos de la tabla 3 permiten observar los promedios de las dimensiones utilizadas en el instrumento CLAS, haciendo un análisis de los datos desde el que mayor porcentaje alcanza tenemos a la Tutoría con un $97.7 \%$ como la dimensión que los estudiantes la responden como la más alta, seguida de la dimensión de Interacción con un $96.8 \%$ como la segunda dimensión respondida por los estudiantes, la tercera dimensión más alta son las Habilidades Sociales con el $94.3 \%$, posteriormente la Heterogeneidad con el $93.7 \%$, a continuación la dimensión de Interdependencia positiva con el $91.7 \%$, en sexto lugar se encuentra la dimensión de Reflexión grupal con el $91.6 \%$, y cierra el cuadro la Evaluación con el $88.4 \%$ de respuesta afirmativa por parte de los estudiantes. Así el cuadro la tabla 4 
muestra el Orden de mayor a menor de las dimensiones, según los porcentajes de afirmación de los estudiantes:

Tabla 4. Orden descendente Dimensiones CLAS

\begin{tabular}{|l|c|}
\hline \multicolumn{1}{|c|}{ Dimensiones } & $\%$ \\
\hline Tutoría & 97.73 \\
\hline Interacción & 96.83 \\
\hline Habilidades Sociales & 94.33 \\
\hline Heterogeneidad & 93.65 \\
\hline Interdependencia positiva & 91.71 \\
\hline Reflexión grupal & 91.61 \\
\hline Evaluación & 88.36 \\
\hline Promedio & 93.46 \\
\hline
\end{tabular}

Gráficamente, los datos se muestran de la siguiente forma:

Figura 1: Gráfico de porcentajes según las dimensiones

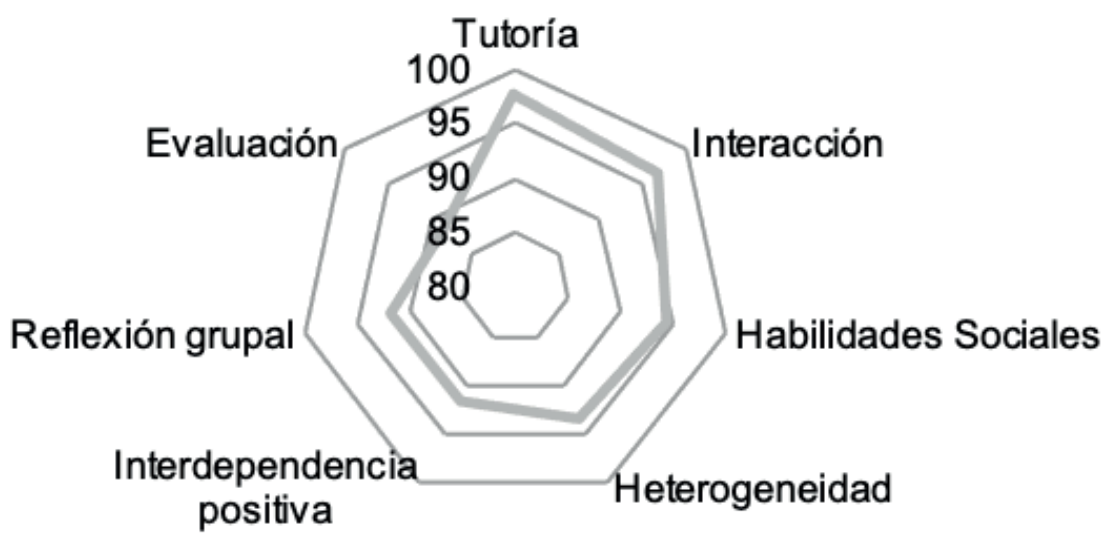

\section{DISCUSIÓN}

La asignatura de Bases de la Comunicación busca generar un espacio conversacional en donde se analicen las formas y métodos por los cuales se puede generar una comunicación eficiente, al hacer uso del AC como herramienta educativa se logra establecer — según los resultados obtenidos - que los objetivos planteados en la asignatura se potencian con el uso de esta metodología, si los estudiantes se manifiestan con tendencia positiva hacia la interacción, la reflexión grupal, la interdependencia positiva, las habilidades sociales, la tutoría, la heterogeneidad y la evaluación, entonces el AC contribuyó a los propósitos de la asignatura generando bases para la comunicación.

A partir de las consideraciones de los estudiantes que han sido rescatadas por medio del instrumento CLAS, el AC permitió en la asignatura de Bases de la Comunicación establecer un precedente metodológico para el aprendizaje con capacidad de aplicación en 
las siguientes cortes de estudiantes, los resultados alcanzados manifiestan un alto nivel de aceptación para con la metodología, lo cual requiere una preparación dedicada de quien la acompaña, y que vendría a ser determinante para alcanzar los resultados obtenidos.

Parecería contradictorio que al hablar de AC la participación del docente sea tan importante, muchas veces daría a pensar que por tratarse de $\mathrm{AC}$ el docente debería tener la mínima participación y aunque uno de los objetivos es alcanzar el aprendizaje autónomo de los grupos de trabajo, sin un correcto apoyo del docente, esto resultaría quimérico, la tutoría implica acompañar el proceso de aprendizaje sin convertirlo en protagonista al docente, pero tampoco desaparecer como tal y abandonar a los educandos, el docente es un observador activo que asiste en la medida que los estudiantes lo necesitan (Johnson y Johnson, 1999), no es quien interfiere sino aquel que fluye en el oleaje y ritmo de los educandos, por esta razón es de vital importancia el previo entrenamiento y reflexión del docente acerca del AC, para el caso el docente necesita conocer la temática a desarrollar, a fin de establecer las técnicas adecuadas dentro del marco del AC, además de los roles, la funcionalidad de los grupos, los acuerdos, y la forma como será evaluado el trabajo de los equipos. Por tal motivo se desatiende cualquier forma de improvisación por parte del docente.

En un estudio de caso realizado por Jauregui, A. et al. (2014), se considera que al desarrollar metodologías cooperativas se requiere, concretamente, de una serie de compromisos en lo que respecta al modo de producir y adquirir los aprendizajes, pues se necesita generar cambios en la concepción del rol del docente y de los estudiantes, en la utilización del espacio y del tiempo y en la relación entre la teoría y la práctica. Según Ruiz, Anguita y Jorrín, (2006) citado en Jauregui, A. et al. (2014): "El aprendizaje cooperativo es esencialmente un proceso solidario de construcción del conocimiento donde los procesos de interacción entre compañeros y compañeras contribuyen a conseguir el éxito en la tarea de todos y cada uno de sus miembros, trabajo que se logra con los acuerdos y valores aceptados por el equipo de trabajo".

Además, se corrobora que los estudiantes logran desarrollar habilidades verbales de interacción, habilidades sociales y de responsabilidad, según León, B. (2014) se determina que: Todos los miembros del equipo comparten la responsabilidad por el aprendizaje. Smith (1996) citado en León, B (2014) destaca como elementos esenciales para que un grupo de aprendizaje cooperativo tenga éxito la interdependencia positiva y la responsabilidad, se han estudiado variables como la composición del equipo, la homogeneidad, heterogeneidad, las competencias que aportan los miembros del equipo, diseño de la tarea e interdependencia, modelos mentales del equipo memoria transactiva, aprendizaje grupal, clima del grupo, cohesión, conflictos, procesos emocionales, comunicación, coordinación, los estudiantes tienen éxito si el equipo también lo tiene, cada miembro del equipo se compromete a realizar su parte del trabajo y se considera al equipo responsable de lograr los objetivos; a partir de estas consideraciones se puede establecer que el aprendizaje cooperativo genera un ambiente de convivencia mejorado en el cual se desenvuelven los educandos. Navarro (2007) afirma que: "aprender cooperativamente representa una ocasión privilegiada para alcanzar objetivos de aprendizaje muy diversos, no solo referidos a los contenidos, sino también orientados al desarrollo de habilidades y destrezas interpersonales, con claros beneficios para el aprendizaje de los alumnos", habilidades y destrezas que pueden ser alineadas al desarrollo de la formación universitaria y para el caso de la asignatura en la cual se trabajó.

EI AC es una herramienta metodológica que depende de muchos factores, no se podría destacar la interacción sin habilidades sociales, o pretender apartar la interdependencia positiva sin un adecuado manejo reflexivo del contexto en donde están los estudiantes, es- 
tos cuatro elementos fortalecen los lazos de convivencia y convierten al proceso de aprendizaje realmente en un accionar de construcción, los estudiantes no solo aprenden contenidos o reflexionan acerca de ellos, los estudiantes, viven el aprendizaje, lo miran desde el comentario de sus iguales, crean un entramado micro social de aprendizaje, de esta forma anulan cualquier tipo de verdad intangible única y abordan la realidad desde el colectivo contrario al individualismo, principalmente fomentando el pensamiento crítico.

Una de las consideraciones del AC es la heterogeneidad, pues mientras más diversos son los grupos de trabajo, más nutrido es el aprendizaje, la heterogeneidad es la base de los demás elementos, si se trabaja con grupos homogéneos se tiene a estandarizar los puntos de observación, no así cuando los estudiantes poseen un disímil que les permite generar reflexiones distintas, este elemento permite fortalecer los restantes elementos: interacción, habilidades sociales, reflexión grupal, interdependencia positiva, la evaluación y la tutoría, y que alcanzan porcentajes elevados en la medición CLAS, según Johnson (1999): "Ios grupos compuestos por estudiantes con diferentes rendimientos y distintos intereses permiten que los alumnos tengan acceso a diversas perspectivas y métodos de resolución de problemas, y producen un mayor desequilibrio cognitivo, necesario para estimular el aprendizaje y el desarrollo cognitivo de los alumnos, lo cual ha sido valorado por los estudiantes participantes del estudio".

Finalmente la evaluación es el elemento ponderado más bajo, como parte del proceso de aprendizaje es necesario solventar estrategias de valoración que vayan desde la evaluación del docente hasta la evaluación autónoma del grupo, durante el proceso de aprendizaje un factor importante es el tiempo de implementación del AC, mientras mayor sea el tiempo de trabajo dentro de la metodología mayor reflexividad y autonomía se debería lograr, se abre así la puerta a nuevos estudios en donde se pueda evidenciar una evaluación consciente y constructiva con orientación hacia la autoevaluación reflexiva como parte de la formación autónoma de quien aprende, para el presente estudio, se siguieron las pautas evaluativas de Jonhson (1999) en las que el docente todavía presenta cierto protagonismo al momento de evaluar tomando pruebas y dando las consignas para generar la evaluación, esto debe irse endosando a los estudiantes a medida que avanza el proceso y se mantiene un mayor periodo de AC hasta alcanzar altos niveles de reflexión grupal, autonomía, hasta que la evaluación también pase a ser su responsabilidad como parte del propósito más alto del AC.

El profundizar en las dimensiones que se valoran en el instrumento CLAS es una de las limitantes, por cuanto cada una de ellas no permite desglosar características adicionales, causales y puntos de observación de los estudiantes, poder conocer el alcance de las dimensiones valoradas por el instrumento, requeriría estudios cualitativos que permitan considerar las apreciaciones de los actores educativos, resultaría interesante complementar este estudio con entrevistas por ejemplo, para que posiblemente la información obtenida alcance mayores niveles de inferencia.

\section{CONCLUSIONES}

Según los resultados obtenidos con el instrumento CLAS, la valoración del aprendizaje cooperativo como herramienta metodológica tiene una aceptación promedio del 93.46 $\%$ entre las siete dimensiones. Para alcanzar este resultado se requiere que quien guía el aprendizaje, así como los materiales y el ambiente estén preparados con la intencionalidad de aplicar el Aprendizaje Cooperativo, gran medida del éxito de esta herramienta radica en haber planificado al detalle cada una de las sesiones enmarcadas en los preceptos del AC. 
El aprendizaje cooperativo potencializa a los equipos de trabajo, para esto, la conformación de los equipos según los criterios permite encaminar a los participantes en el desarrollo de habilidades sociales que durante un proceso de educación tradicional se suceden limitadas por el rol activo del profesor y pasivo de los estudiantes.

La escala de aplicación CLAS se convierte en un instrumento útil para quienes hacen uso del AC, a fin de conocer una forma de valoración de los estudiantes que han sido participantes en el proceso de aplicación de las técnicas del AC, lo cual ayuda a objetivizar dicha aplicación y resulta recomendable.

\section{REFERENCIAS}

Atxurra, C. V.-G. (2015). Diseño y Validación de la Escala de Aplicación del Aprendizaje Cooperativo (CLAS). Deusto: Revista Psicodidáctica 20 (2): 339-357.

Bouzas, P. (2002). Repercusión del aprendizaje cooperativo sobre el rendimiento y desarrollo personal y social de los estudiantes. España: Revista de ciencias de la educación 192: 505-522.

Díaz Aguado, M. (2004). Cómo mejorar la convivencia escolar ante los retos de la educación del siglo XXI. España: Foro de la convivencia en los centros educativos. Educrea 1-40.

Fernández, E. (2010). El trabajo en equipo mediante el Aprendizaje Cooperativo. Valencia - España: Departamento de Psicología Evolutiva y de la Educación.

Gómez, I. F., Agut, A. y Franch J. (2003). Cómo redactar un artículo científico. Revista AVEPA. 167-173.

Jauregui, P. V. (2014). Estudio de caso y aprendizaje cooperativo en la universidad. Revista de Curriculum y Formación del profesorado, 18 (1): 413-429.

Johnson, D. J. (1999). El aprendizaje cooperativo en el aula. Buenos Aires, Argentina: Paidós Ibérica.

León del Barco, B. C. (2014). Determinantes en la eficacia del aprendizaje cooperativo, una experiencia en el EEES. Revista de Investigación Educativa. Recuperado de https://doi.org/10.6018/ rie.32.2.172721.

Loor, L. P. (2018). El aprendizaje cooperativo como una estrategia de enseñanza del Idioma Onglés en las escuelas públicas del Ecuador. Revista Científica Dominio de las Ciencias Recuperado de https://dialnet.unirioja.es/descarga/articulo/6560189.pdf.

Navarro, L. (2007). El aprendizaje cooperativo. Madrid, España: PPC. EDUCAR.

Placencia, Z. y (2015). El aprendizaje cooperativo como estrategia didáctica para enseñar estudios sociales a los estudiantes de octavo año de EGB de la Unidad Educativa Sinincay. Cuenca, Ecuador: Universidad Politécnica Salesiana. Recuperado de https://dspace.ups.edu.ec/bitstream/123456789/8782/1/UPS-CT005004.pdf. 